\title{
Effect of fecal microbiota transplantation on experimental colitis in mice
}

\author{
JULAN ZHOU $^{1}$, ZHONGYIN ZHOU ${ }^{1}$, PANPAN JI ${ }^{2}$, MIN MA $^{1}$, JINKUN GUO ${ }^{1}$ and SHUJUAN JIANG ${ }^{1}$ \\ ${ }^{1}$ Department of Gastroenterology, Renmin Hospital of Wuhan University, Hubei Key Laboratory of Digestive System Disease, \\ Wuhan, Hubei 430060; ${ }^{2}$ Department of Gastroenterology, Zhengzhou Third People's Hospital, Zhengzhou, \\ Henan 450000, P.R. China
}

Received September 5, 2018; Accepted February 6, 2019

DOI: $10.3892 /$ etm.2019.7263

\begin{abstract}
The aim of the present study was to investigate the effect of fecal microbiota transplantation (FMT) on the acute inflammatory response in a murine model of dextran sulfate sodium (DSS)-induced colitis, and to delineate the putative underlying mechanism(s). Mice were divided into four groups, namely the normal control, DSS, 5-aminosalicylic acid (5-ASA) and FMT group. Mice in the DSS, 5-ASA and FMT groups were orally administered 3\% DSS (w/v) solution for 7 days to induce colitis. On days 1, 3, 5 and 7, mice in the DSS, 5-ASA and FMT groups were respectively administered $0.5 \%$ carboxymethylcellulose sodium, 5-ASA suspension and fecal suspension by enema. The disease activity index of each mouse was calculated on a daily basis. All mice were sacrificed on day 8 , and the length of their colons was measured. Myeloperoxidase (MPO) activity, and the levels of tumor necrosis factor $\alpha$ (TNF- $\alpha$ ), interleukin (IL)-1 $\beta$ and IL-10 in the colon tissues of each group were also measured. Compared with that in the DSS group, FMT ameliorated the severity of inflammation due to ulcerative colitis in mice, which was accompanied by a significantly decreased MPO activity, reduced levels of TNF- $\alpha$ and IL-1 $\beta$, and an increased level of IL-10 in colon tissue (all $\mathrm{P}<0.05$ ). Taken together, these results demonstrated that FMT exerted a therapeutic effect on experimental colitis in mice, and the associated mechanism is likely to involve the remodeling of the intestinal flora and regulation of intestinal T-cell immunity homeostasis.
\end{abstract}

\section{Introduction}

Fecal microbiota transplantation (FMT) is a treatment method that transfers the functional bacteria in human feces into the

Correspondence to: Professor Zhongyin Zhou, Department of Gastroenterology, Renmin Hospital of Wuhan University, Hubei Key Laboratory of Digestive System Disease, 238 Jiefang Road, Wuhan, Hubei 430060, P.R. China

E-mail: wuyangwu712@163.com

Key words: fecal microbiota transplantation, intestinal symbiotic flora, ulcerative colitis, immunity homeostasis, dysbacteriosis gastrointestinal tract of patients through different routes of administration, so as to remodel the intestinal flora structure, thereby achieving the purpose of treating intestinal-associated diseases $(1,2)$. Inflammatory bowel disease (IBD) is a disease characterized by recurrent episodes of chronic inflammation of the intestine, and the term is predominantly used in reference to two conditions: Ulcerative colitis (UC) and Crohn's disease. At present, the pathological mechanism of IBD remains to be fully elucidated, although the important role of intestinal symbiotic bacteria and flora disorders in the pathogenesis of IBD has been widely investigated (3). Numerous studies have demonstrated that the numbers of intestinal dominant bacteria, or anti-inflammatory bacteria (including firmicutes, lactobacilli and bacteroides) of patients with IBD are reduced, whereas those of harmful bacteria, including enterococcus and proteobacteria, are increased, and the intestinal microbial diversity is markedly reduced (4). Therefore, adjusting intestinal flora disturbances and restoring the homeostasis between host and intestinal microorganisms has become a novel strategy for the treatment of IBD.

A large number of clinical trials have confirmed that FMT is a safe and effective method for the treatment of recurrent Clostridium difficile infections, and a recent review of IBS by El-Salhy and Mazzawi (5) revealed that FMT in patients with IBS led to a reversion of the gut microbiota dysbiosis to normalcy, reduced the symptoms of IBS in $\sim 70 \%$ of patients and was not associated with any serious adverse events. FMT was therefore demonstrated to be a promising tool for managing IBS. It appears to be an effective, easy and inexpensive procedure (5); however, studies on the treatment of IBD have been limited to partial case reports and studies, and the specific mechanism of the anti-inflammatory effect remains elusive (6). A previous study that established an experimental UC model in mice with dextran sulfate sodium (DSS) revealed an intestinal flora disturbance similar to that observed in human IBD (7). The animal model of FMT is an invaluable complement to the clinical study of the efficacy of FMT due to the controllability of various variables.

Therefore, the aim of the present study was to investigate the effect of FMT on the acute inflammatory response in a murine model of DSS-induced colitis, and to attempt to elucidate the possible underlying mechanism(s). The results obtained indicate that FMT may exert a therapeutic effect on 
experimental colitis in mice via reshaping the intestinal flora and regulating intestinal T-cell immunity homeostasis.

\section{Materials and methods}

Experimental animals. A total of $32 \mathrm{BALB} / \mathrm{c}$ mice (age, 8 weeks) were used for the present study (other characteristics: Female; average weight, $21.0 \pm 3.0 \mathrm{~g}$ ). The study also employed, as FMT donor animals, 3 Sprague Dawley (SD) rats (female; weight, 200-220 g; age, 8 weeks) and 3 C57/BL6 mice (female; age, 8 weeks).

All of the animals were purchased from the Experimental Animal Center of Wuhan University (Wuhan, China) and raised in the specific pathogen-free-grade animal room of the Stomatological Hospital of Wuhan University (Wuhan, China; certificate no. 2800360003056) with adaptive feeding for 1 week prior to the start of the experiment. The room temperature was maintained at $20 \pm 2^{\circ} \mathrm{C}$, with a relative humidity of $50 \pm 10 \%$ and artificial lighting (12-h light/dark cycle). All procedures for the care and handling of animals in the present study were approved by the University of Wuhan Animal Care Committee [certificate no. SYXK (E) 2009-0027].

Reagents. DSS (molecular weight, 36,000-50,000 Da) was purchased from MP Biomedicals, LLC (Santa Ana, CA, USA), 5-aminosalicylic acid (5-ASA) was from Maya Reagent Co., Ltd. (Jiaxing, China); and sodium carboxymethylcellulose was obtained from Sinopharm Chemical Reagent Co., Ltd. (Shanghai, China). The stool occult blood test kit (cat. no. 32365192910) and the kit (cat. no. 30365193913) for the determination of myeloperoxidase (MPO) activity were purchased from the Nanjing Jiancheng Bioengineering Institute (Nanjing, China). An ELISA kit (cat. no. 35790106219) for detecting the levels of tumor necrosis factor- $\alpha$ (TNF- $\alpha$ ), interleukin (IL)- $1 \beta$ and IL-10 was obtained from XinBosheng Biotechnology Co., Ltd. (Xi'an, China).

Animal groups and treatment. The mice were randomly divided into 4 groups by using the numerical table method, with 8 mice in each group, namely the i) normal group; ii) DSS group; iii) 5-ASA group; and iv) FMT group. From the first day of the experiment, with the exception of the normal group, the other 3 groups were administered a $3 \%$ solution of DSS provided as their freely provided drinking water for 7 days, in order to induce the acute UC model, and the DSS-containing water was replaced daily. On days 1,3,5 and 7, the mice in the DSS, 5-ASA and FMT groups were respectively given $200 \mu \mathrm{l}$ $0.5 \%$ carboxymethylcellulose sodium, 5-ASA in suspension $(100 \mathrm{mg} / \mathrm{kg})(8)$ or $200 \mu \mathrm{l}$ fecal suspension by enema. By contrast, the normal group was left untreated and provided with normal food and water ad libitum. On the eighth day of the experiment, the mice were sacrificed by cervical dislocation following anesthesia, and the abdominal cavity was subsequently dissected, the colon was isolated and its length was measured.

Intestinal cavities were cut along the vertical axis of the mesentery, and after rinsing with ice-cold PBS, three portions of the tissue were retained from an identical part for each mouse, of which the lesioned sections of $\sim 0.4 \mathrm{~cm}$ were fixed with $4 \%$ paraformaldehyde, embedded with paraffin, sliced and subjected to $\mathrm{H} \& \mathrm{E}$ staining. The two remaining parts of the colon tissue were immediately placed in liquid nitrogen and subsequently transferred to a refrigerator at $-80^{\circ} \mathrm{C}$ prior to biochemical testing.

Pharmaceutical preparations. In order to prepare the 5-ASA suspension, 5 -ASA was added to $0.5 \%$ sodium carboxymethyl cellulose $(5 \mathrm{ml})$, followed by vortexing, to obtain a $20 \mathrm{mg} / \mathrm{ml}$ suspension for subsequent use. This mixing step was performed prior to performing each enema.

For the preparation of the fecal bacteria liquid (9), SD rats and C57/BL6 mice were sacrificed by cervical dislocation following anesthesia, and the cecum contents were extracted from the 6 donor animals. After weighing the extracted cecum contents in $3 \mathrm{ml} / \mathrm{g}$ sodium carboxymethyl cellulose solution, stirring on ice and vortex-mixing, the contents were placed in a clean centrifuge tube and centrifuged at 5,000 x g for $20 \mathrm{~min}$ at $20^{\circ} \mathrm{C}$. The supernatant was then taken and placed in a refrigerator at $-80^{\circ} \mathrm{C}$ for cryopreservation.

Mice enema method. A solution of $4 \%$ chloral hydrate $(0.4 \mathrm{mg} / \mathrm{kg}$ body weight) was injected intraperitoneally into the mice to induce mild anesthesia. The mice were placed into the prone position, followed by placement of a plastic tube (inner diameter, $2 \mathrm{~mm}$ ). After applying paraffin oil onto its surface, the tube was gently inserted into the colon through the anus of the mice up to $\sim 4 \mathrm{~cm}$ from the top of the tube to the anus, when the suspension was warmed to body temperature and the fecal bacteria solution $(200 \mu \mathrm{l})$ was slowly injected. Other suspensions were similarly administered. Subsequently, the tube was withdrawn and a cotton swab was pressed onto the anus. By clasping their tails, the mice were held upside down for 1 min, prior to placing the mice back into the cage. Mice in the normal group received no treatment.

Observation indexes. Normal functions of the mice, including feeding and activity, weight, fecal composition and fecal occult blood of mice, were monitored daily, and the disease activity index (DAI) was determined. The DAI score was calculated as follows: 0 points for no weight loss, 1 point for a $1-5 \%$ reduction in weight; 2 points for a decrease in weight of $5-10 \% ; 3$ points for a loss in weight of $10-15 \%$; and 4 points for a decrease of $>15 \%$ in weight. Scores for the condition of the stools were as follows: 0 points, normal (formalized) stools; 2 points, loose stools (mushy, semi-formed stools that did not adhere to the anus); 4 points, loose stools (may adhere to the anus in the water sample). In addition, 0 points were assigned if no fecal occult blood or gross blood feces were identified; and a positive fecal occult blood test scored 2 points, whereas gross blood count was credited with 4 points. The score for the above three characteristics were added together to obtain the DAI for each mouse, and the severity of colitis was thereby determined (10).

The degree of histological damage was assessed by determining the product of the scores of inflammation, lesion depth, crypt destruction and lesion range. The average value was used as the score of histological damage of the colon (11).

Determination of the MPO activity in colon tissue. The tissue weight was accurately determined, and the colon tissue 
homogenate medium was added at a weight-to-volume ratio of 1:19. The mixture was fully homogenized on ice to obtain a $5 \%$ homogenate, and the remainder of the steps were performed according to the instructions of the kit (obtained from the Nanjing Jiancheng Bioengineering Institute).

ELISA assay for determination of TNF- $\alpha, I L-1 \beta$ and IL-10. The tissue weight was accurately determined and pre-cooled PBS was added at a weight-to-volume ratio of 1:9. A sufficient amount of homogenate was placed on ice and subjected to ultrasonic crushing in parallel. Finally, the slurry was centrifuged at $10,000 \times \mathrm{g}$ at $4^{\circ} \mathrm{C}$ for $5 \mathrm{~min}$, and the supernatant was retained for detection of the contents of the various cytokines, according to the instructions provided by the ELISA kit.

Statistical methods. SPSS version 17 software (SPSS, Inc., Chicago, IL, USA) was used for data analysis. Values are expressed as the mean \pm standard deviation. Comparisons between multiple groups were performed by analysis of variance and the least-significance difference test. $\mathrm{P}<0.05$ was considered to indicate a statistically significant difference.

\section{Results}

FMT attenuates $U C$-induced reduction in colonic length. The colonic length in the DSS, 5-ASA and FMT groups was identified to be shorter compared with that in the normal group $(\mathrm{P}<0.05)$, the length in the 5-ASA and the FMT groups was greater than that in the DSS group $(\mathrm{P}<0.05)$. No significant difference in colonic length was identified between the 5-ASA and the FMT groups ( $\mathrm{P}>0.05$, Fig. 1).

Weight changes in each group. Prior to the experiment, no significant differences in body weight were observed between the groups $(\mathrm{P}>0.05)$. At the end of the experiment, however, the weight of the mice in the DSS, 5-ASA and FMT groups had clearly decreased. Furthermore, the total weight loss in these 3 groups (difference between the start and end of the experiment) was significantly different compared with that in the normal group $(\mathrm{P}<0.05)$. Compared with that in the DSS group, the weight loss in the 5-ASA group and FMT group was significantly decreased ( $\mathrm{P}<0.05$, Fig. 2$)$.

Histological injury score of mice in each group. In the DSS, 5-ASA and FMT groups, colonic mucosal epithelial cells were widely absent, glands had lost their integrity, an extensive infiltration of inflammatory cells was apparent and typical inflammatory reactions were observed (Fig. 3). The histological scores for these 3 groups were higher compared with those in the normal control group, and the difference was statistically significant $(\mathrm{P}<0.05)$. The histological score in the FMT group was lower compared with that in the DSS group $(\mathrm{P}<0.05)$, but this difference was less pronounced than that observed between the 5-ASA and the DSS groups (Fig. 4).

DAI score in each group. In the group subjected to FMT treatment, the body weight loss, fecal consistency and occult blood status were improved in the FMT group compared with those in the DSS group $(\mathrm{P}<0.05)$. Furthermore, the DAI score was higher compared with that in the 5-ASA group, although

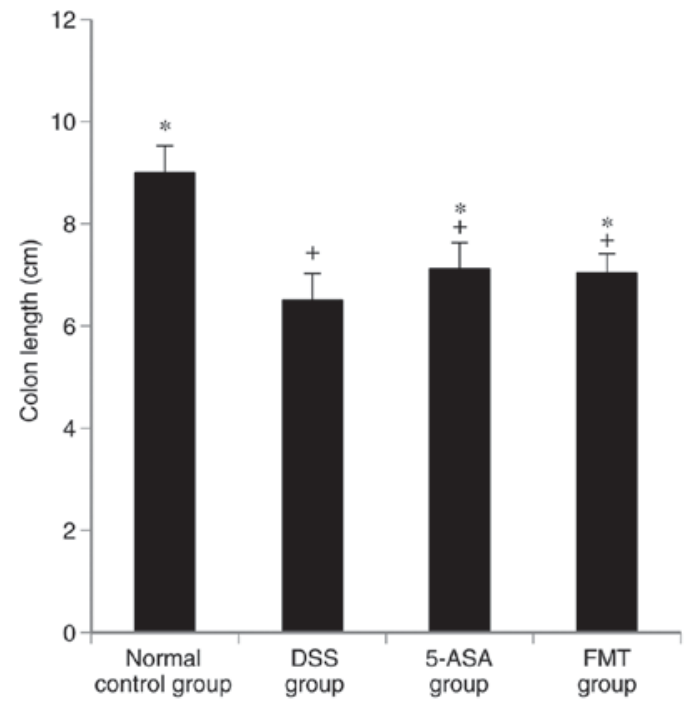

Figure 1. Colon length (in $\mathrm{cm}$ ) of the mice in each group. Values are expressed as the mean \pm standard deviation. ${ }^{*} \mathrm{P}<0.05$ vs. DSS group. ${ }^{+} \mathrm{P}<0.05$ vs. normal group. DSS, dextran sulfate sodium; 5-ASA, 5-aminosalicylic acid; FMT, fecal microbiota transplantation.

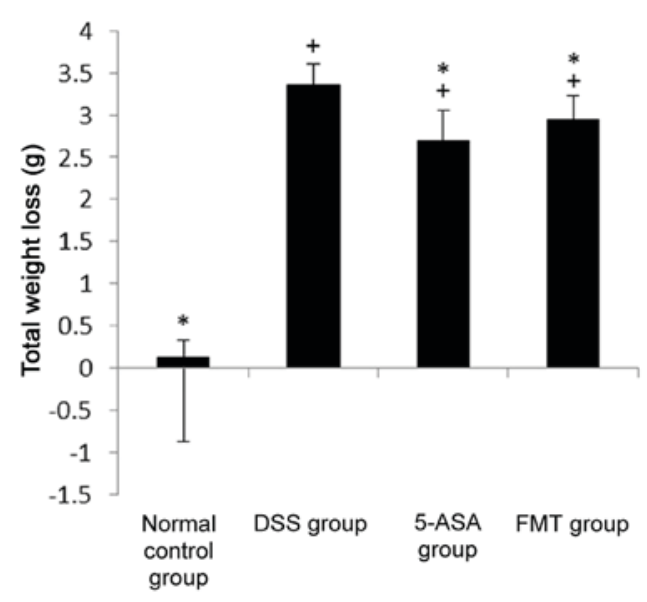

Figure 2. Total weight loss (in g) of mice in each group. Values are expressed as the mean \pm standard deviation. ${ }^{*} \mathrm{P}<0.05$ vs. DSS group. ${ }^{+} \mathrm{P}<0.05$ vs. normal group. DSS, dextran sulfate sodium; 5-ASA, 5-aminosalicylic acid; FMT, fecal microbiota transplantation.
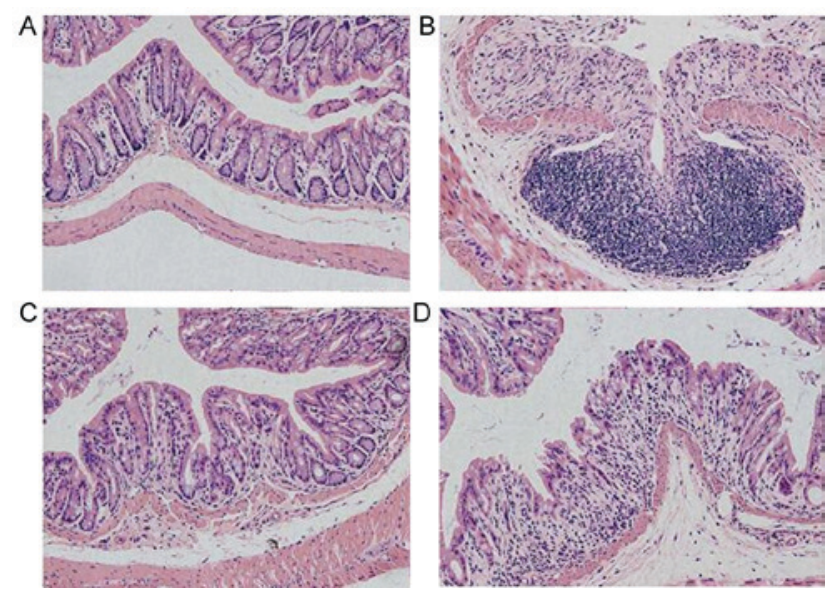

Figure 3. H\&E staining of colonic tissue of mice in each group (magnification, x400). (A) Normal group; (B) dextran sulfate sodium group; (C) 5-aminosalicylic acid group; (D) fecal microbiota transplantation group. 


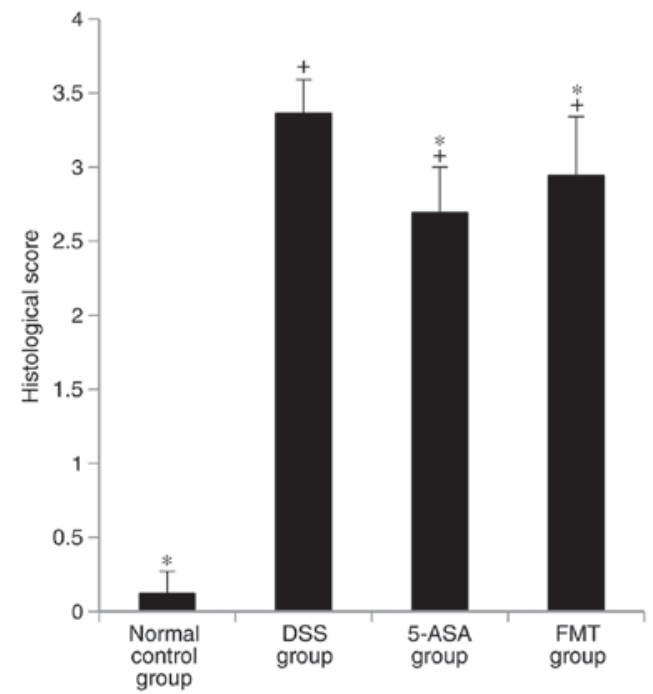

Figure 4. Histological scores of the mice in each group. Endoscopic measurements of each experimental group were evaluated using histological grading scores. Values are expressed as the mean \pm standard deviation. " $\mathrm{P}<0.05$ vs. DSS group. ${ }^{+} \mathrm{P}<0.05$ vs. normal group. DSS, dextran sulfate sodium; 5-ASA, 5-aminosalicylic acid; FMT, fecal microbiota transplantation.

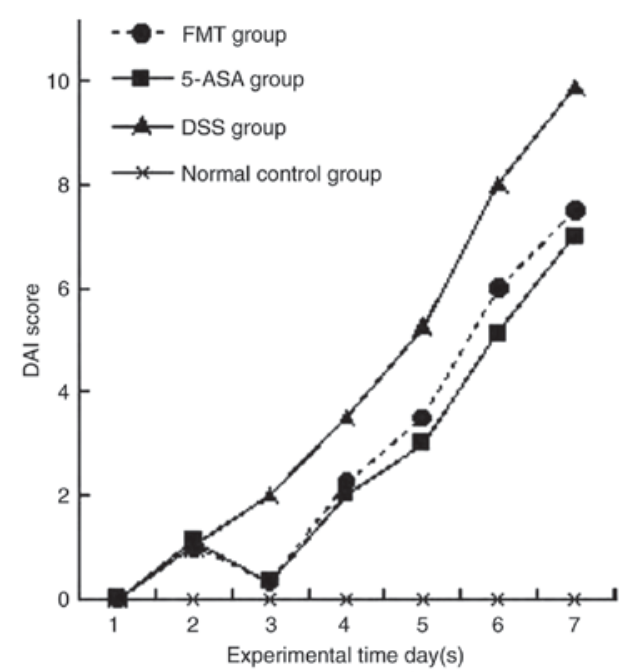

Figure 5. DAI score of mice in each group. DSS, dextran sulfate sodium; 5-ASA, 5-aminosalicylic acid; FMT, fecal microbiota transplantation; DAI, disease activity index.

the difference was revealed not to be statistically significant ( $\mathrm{P}>0.05$, Fig. 5). It is noteworthy that the DAI score recorded for the experiment on day 3 was lower than that determined on day 2 . The possible explanation for this was that no enema stress occurred on the second day, and the mice gained weight after having had a 24-h opportunity to feed undisturbed.

Colonic MPO activity, and the levels of TNF- $\alpha, I L-1 \beta$ and $I L-10$. MPO activity, and the levels of TNF- $\alpha$ and IL- $1 \beta$ in the DSS, 5-ASA and FMT groups were significantly higher compared with those in the normal group $(\mathrm{P}<0.05)$, while the level of IL-10 was lower than that in the normal group $(\mathrm{P}<0.05)$. MPO activity, and the levels of TNF- $\alpha$ and IL-1 $\beta$ in the 5-ASA and FMT group were lower compared with those in the DSS group, and these differences were shown to be

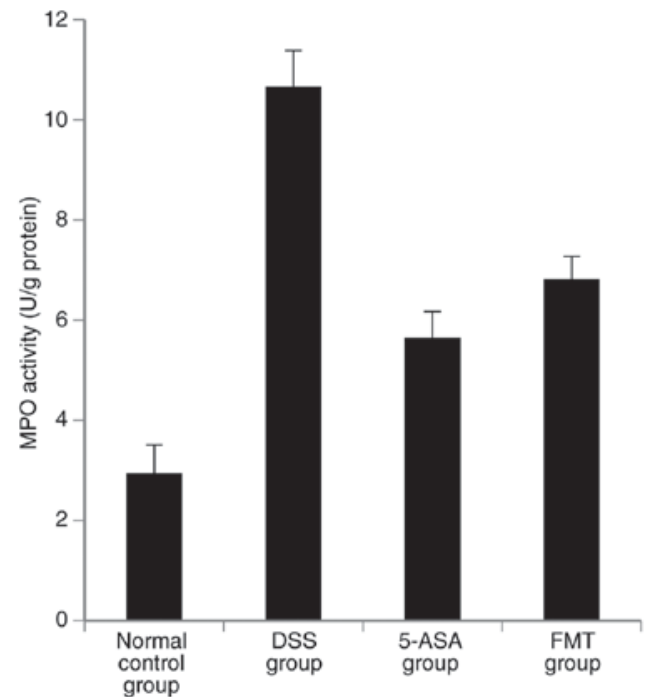

Figure 6. MPO activity levels in colonic tissue of mice in each group. The MPO activity in the DSS group, the 5-ASA group and the FMT group was significantly higher than that in the normal group $(\mathrm{P}<0.05)$. Furthermore, the MPO activity in the FMT group was lower than that in the DSS group, and the difference was statistically significant $(\mathrm{P}<0.05)$. Values are expressed as the mean \pm standard deviation. MPO, myeloperoxidase; DSS, dextran sulfate sodium; 5-ASA, 5-aminosalicylic acid; FMT, fecal microbiota transplantation.

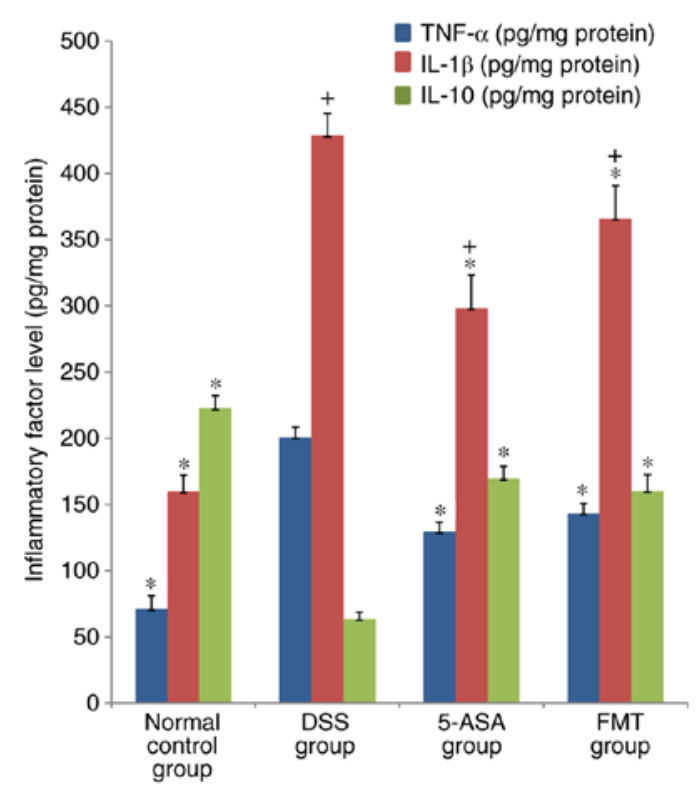

Figure 7. TNF- $\alpha$, IL-1 $\beta$ and IL-10 levels in colonic tissue of mice in each group determined by ELISA. Values are expressed as the mean \pm standard deviation. ${ }^{*} \mathrm{P}<0.05$ vs. DSS group. ${ }^{+} \mathrm{P}<0.05$ vs. normal group. TNF- $\alpha$, tumor necrosis factor $\alpha$; IL, interleukin; DSS, dextran sulfate sodium; 5-ASA, 5-aminosalicylic acid; FMT, fecal microbiota transplantation.

statistically significant $(\mathrm{P}<0.05)$; in addition, the level of IL-10 was significantly higher compared with that in the DSS group $(\mathrm{P}<0.05$; Figs. 6 and 7$)$.

\section{Discussion}

In the present study, a murine model of acute UC was induced by the administration of 3\% DSS solution, and the efficacy of FMT to reduce intestinal inflammation in the constructed 
UC model was preliminarily assessed. It was revealed that the weight loss, stool consistency, fecal occult blood, colon length change and intestinal mucosal injury in mice treated with FMT were all improved compared with those in the model group. In the FMT group, the MPO activity in colon tissues was decreased, and the levels of the inflammatory factors TNF- $\alpha$ and IL-1 $\beta$ were also decreased, whereas the level of the immunomodulator IL-10 was increased compared with that in the DSS group, although this was not as effective as treatment with 5-ASA.

Germ-free mice are more likely to develop experimental UC upon DSS induction, and this phenomenon was previously demonstrated to be reversed following fecal bacteria transplantation from healthy wild mice, indicating that intestinal symbiotic bacteria are able to slow down the formation of DSS-induced UC to a certain extent (12). In addition, an increasing number of studies suggest that an imbalance in the number and function of type 1 T-helper cells (Th1)/Th17 and forkhead box $\mathrm{P}^{+}$regulatory $\mathrm{T}$ cells (Treg) may cause intestinal immune dysfunction and immunopathological damage, thereby leading to IBD (13). In the intestinal tract, Th1 cells predominantly secrete the pro-inflammatory cytokines interferon- $\gamma$ and TNF- $\alpha$. Th17 cells, a subgroup of T cells, secrete the pro-inflammatory cytokine IL-17. Treg cells secrete the anti-inflammatory factor IL-10 and exert a protective role in IBD (14). Once the symbiotic balance between host-intestinal microbes and the immune system is broken, inflammatory diseases including IBD are able to arise. The occurrence of intestinal inflammation is caused by the overly strong immune response in susceptible individuals induced by an imbalance of the intestinal flora. Certain studies have indicated that FMT is able to reconstruct the composition of the intestinal flora in animal models (15).

Recently, a number of studies have focused on determining which types of symbiotic bacteria inhibit inflammation and investigating the associated, underlying mechanisms. It has been indicated that certain intestinal symbiotic bacteria and their metabolites are able to promote the immune balance between Th cells and Treg cells in mammals, thereby preventing, or effectively treating, IBD. Fragile Pseudomonas bacilli and its polysaccharide $A$ are able to inhibit the infiltration of neutrophils in the intestinal tract of experimental animals with IBD by stimulating Treg cells to produce IL-10, which leads to a reduction in the release of cytokines including TNF- $\alpha$, IL-1 $\beta$ and IL-23 (16). It was also confirmed that Sporogenous sclerenchyma of fusobacteria IV and XIVa may allay DSS-induced UC in mice, where the action of the bacteria was facilitated by inducing Treg cells (17). Fragile Clostridium is able to reduce 2,4,6-trinitrobenzene sulfonic acid-induced colitis. In addition, short-chain fatty acids, produced by intestinal symbiotic bacteria, are able to regulate the homeostasis of Treg cells in the colon, which is another anti-inflammatory mechanism occurring in the gut microbiota (18). In the present study, UC was induced in healthy mice by DSS provided with drinking water, which was alleviated by enema administration of FMT, and a mixture of fecal bacteria from rats and mice was used to increase the microbial diversity. This led to a remodeling of the flora diversity, and it is possible to hypothesize that, to a certain extent, various microbes and associated metabolites may adjust the intestinal mucosal immune status, including the balance between Treg and Th cells, based on the promotion of secretion of anti-inflammatory cytokines, e.g. IL-10, and inhibition of the secretion of the pro-inflammatory cytokines TNF- $\alpha$, IL-1 $\beta$ and IL-17, such as to reduce inflammation and repair the intestinal mucosal barrier function.

In the present study, FMT was applied to the lower gastrointestinal tract by enema, which led to certain therapeutic effects. However, various issues remain to be addressed. For instance, the identity of the specific microorganism(s) or metabolite(s) that are able to exert the anti-inflammatory effects. Furthermore, it remains elusive whether the intestinal flora imbalance is the cause or the result of IBD, and the most effective viscosity and transplantation volume of the fecal bacteria solution remains to be determined. The effects of the administration of FMT in animal models may be further analyzed with the application of $16 \mathrm{~S}$ ribosomal RNA gene detection technology to investigate the intestinal microorganism macrogenome, in order to elucidate the relevant mechanism(s) underlying the effects of FMT in the treatment of IBD. The production of microbiotic preparations for individualized treatment may hold great promise for the clinical treatment of IBD. However, one limitation of the present study was that mice in the normal group received no treatment.

In conclusion, the present study suggested that FMT was efficient in treating DSS-induced colitis in mice. DSS is one of the most common pharmacological drugs that is used to induce experimental colitis and closely simulates the clinical pathological manifestations of human UC. Of note, no significant differences in the therapeutic effects between 5-ASA and FMT in UC were identified in the present study. However, 5-ASA was slightly more efficient regarding the improvement of certain parameters, such as reducing the levels of inflammatory factors and upregulation of anti-inflammatory factors. Furthermore, by remodelling the intestinal flora, FMT may restore the immune balance that depends on the interaction between the host and symbiotic microorganisms, to inhibit the release of pro-inflammatory cytokines and promote the production of anti-inflammatory cytokines, so as to reduce the inflammation in the colon.

\section{Acknowledgements}

Not applicable.

\section{Funding}

The present study was supported by the Scientific and Technological Project of Hubei Province (grant no. 2015BKB013) and the Natural Science Foundation of Hubei Province in China (grant no. 2013CHB025).

\section{Availability of data and materials}

The datasets used and/or analyzed during the current study are available from the corresponding author on reasonable request.

\section{Authors' contributions}

$\mathrm{ZZ}$ conceived and designed the experiments. PJ performed the experiments. MM, SJ and JG were responsible for analysis 
and interpretation of data, statistical analysis and manuscript drafting, and provided critical revisions of the manuscript for important intellectual content. JZ was responsible for the research creation and design, analysis and interpretation of data, and manuscript drafting, and provided critical revision of the manuscript for important intellectual content. All authors read and approved the final manuscript.

\section{Ethics approval and consent to participate}

All procedures for the care and handling of animals used in the present study were approved by the University of Wuhan Animal Care Committee [Wunah, China; certificate no. SYXK (E) 2009-0027].

\section{Patient consent for publication}

Not applicable.

\section{Competing interests}

The authors declare that they have no competing interests.

\section{References}

1. Li D, Wang P, Wang P, Hu X and Chen F: The gut microbiota: A treasure for human health. Biotechnol Adv 34: 1210-1224, 2016.

2. Yadav V, Varum F, Bravo R, Furrer E, Bojic D and Basit AW: Inflammatory bowel disease: Exploring gut pathophysiology for novel therapeutic targets. Transl Res 176: 38-68, 2016.

3. Miyoshi J and Chang EB: The gut microbiota and inflammatory bowel diseases. Transl Res 179: 38-48, 2017.

4. Morgan XC, Tickle TL, Sokol H, Gevers D, Devaney KL, Ward DV, Reyes JA, Shah SA, LeLeiko N, Snapper SB, et al: Dysfunction of the intestinal microbiome in inflammatory bowel disease and treatment. Genome Biol 13: R79, 2012.

5. El-Salhy M and Mazzawi T: Fecal microbiota transplantation for managing irritable bowel syndrome. Expert Rev Gastroenterol Hepatol 12: 439-445, 2018.

6. Wohlgemuth S, Haller D, Blaut M and Loh G: Reduced microbial diversity and high numbers of one single Escherichia coli strain in the intestine of colitic mice. Environ Microbiol 11: 1562-1571, 2009.
7. Kaur R, Thakur S, Rastogi P and Kaushal N: Resolution of Cox mediated inflammation by Se supplementation in mouse experimental model of colitis. PLoS One 13: e0201356, 2018.

8. Moulari B, Pertuit D, Pellequer Y and Lamprecht A: The targeting of surface modified silica nanoparticles to inflamed tissue in experimental colitis. Biomaterials 29: 4554-4560, 2008.

9. Hamilton MJ, Weingarden AR, Sadowsky MJ and Khoruts A: Standardized frozen preparation for transplantation of fecal microbiota for recurrent Clostridium difficile infection. Am J Gastroenterol 107: 761-767, 2012.

10. Cooper HS, Murthy SN, Shah RS and Sedergran DJ: Clinicopathologic study of dextran sulfate sodium experimental murine colitis. Lab Invest 69: 238-249, 1993.

11. Murano M, Maemura K, Hirata I, Toshina K, Nishikawa T, Hamamoto N, Sasaki S, Saitoh O and Katsu K: Therapeutic effect of intracolonically administered nuclear factor kappa B (p65) antisense oligonucleotide on mouse dextran sulphate sodium (DSS)-induced colitis. Clin Exp Immunol 120: 51-58, 2000.

12. Maslowski KM, Vieira AT, Ng A, Kranich J, Sierro F, Yu D, Schilter HC, Rolph MS, Mackay F, Artis D, et al: Regulation of inflammatory responses by gut microbiota and chemoattractant receptor GPR43. Nature 461: 1282-1286, 2009.

13. Maloy KJ and Powrie F: Intestinal homeostasis and its breakdown in inflammatory bowel disease. Nature 474: 298-306, 2011.

14. Shale M, Schiering C and Powrie F: CD4(+) T-cell subsets in intestinal inflammation. Immunol Rev 252: 164-182, 2013.

15. Manichanh C, Reeder J, Gibert P, Varela E, Llopis M, Antolin M, Guigo R, Knight R and Guarner F: Reshaping the gut microbiome with bacterial transplantation and antibiotic intake. Genome Res 20: 1411-1419, 2010.

16. Mazmanian SK, Round JL and Kasper DL: A microbial symbiosis factor prevents intestinal inflammatory disease. Nature 453: 620-625, 2008

17. Atarashi K, Tanoue $\mathrm{T}$, Shima $\mathrm{T}$, Imaoka A, Kuwahara $\mathrm{T}$, Momose Y, Cheng G, Yamasaki S, Saito T, Ohba Y, et al: Induction of colonic regulatory $\mathrm{T}$ cells by indigenous clostridium species. Science 331: 337-341, 2011.

18. Smith PM, Howitt MR, Panikov N, Michaud M, Gallini CA, Bohlooly-Y M, Glickman JN and Garrett WS: The microbial metabolites, short-chain fatty acids, regulate colonic Treg cell homeostasis. Science 341: 569-573, 2013.

This work is licensed under a Creative Commons Attribution-NonCommercial-NoDerivatives 4.0 International (CC BY-NC-ND 4.0) License. 